\title{
Preventable anesthesia mishaps: a study of human factors*
}

\author{
Jeffrey B Cooper, Ronald S Newbower, Charlene D Long, Bucknam McPeek
}

Qual Saf Health Care 2002;11:277-283

See end of article for authors' affiliations

A modified critical-incident analysis technique was used in a retrospective examination of the characteristics of human error and equipment failure in anesthetic practice. The objective was to uncover patterns of frequently occurring incidents that are in need of careful prospective investigation. Forty seven interviews were conducted with staff and resident anesthesiologists at one urban teaching institution, and descriptions of 359 preventable incidents were obtained. Twenty three categories of details from these descriptions were subjected to computer-aided analysis for trends and patterns. Most of the preventable incidents involved human error $(82 \%)$, with breathing-circuit disconnections, inadvertent changes in gas flow, and drug syringe errors being frequent problems. Overt equipment failures constituted only $14 \%$ of the total number of preventable incidents, but equipment design was indictable in many categories of human error, as were inadequate experience and insufficient familiarity with equipment or with the specific surgical procedure. Other factors frequently associated with incidents were inadequate communication among personnel, haste or lack of precaution, and distraction. Results from multi-hospital studies based on the methodology developed could be used for more objective determination of priorities and planning of specific investments for decreasing the risk associated with anesthesia.

$\mathrm{P}$ reventable mishaps resulting from human error contribute to anesthetic risk. Yet, the specific etiology of the contribution has received little attention. Even estimates of the magnitude of the contribution of error to anesthetic mortality vary. ${ }^{1}$ Human errors were believed to be a factor in $87 \%$ of 80 deaths attributable to anesthesia reported by Dripps et $a l^{2}$ in $65 \%$ of 52 deaths reported by Clifton and Hotten, ${ }^{3}$ and in $83 \%$ of 589 deaths reported by Edwards et al. ${ }^{4}$ In the BeecherTodd study, $7.5 \%$ of the deaths reported were attributed to "gross anesthetic mismanagement". These and other risk studies have concentrated primarily on quantitating overall anesthetic risk, using mortality as the one measure of negative outcomes. Attempts to identify risk factors have usually encompassed only those variables directly associated with operative procedures or the patient's disease, age, physical status, etc. Factors associated with anesthetists and/or factors that may have predisposed anesthetists to err have, with a few exceptions, ${ }^{6-8}$ not been analyzed. Furthermore, no study has focused on the process of error-its causes, the circumstances that surround it, or its association with specific procedures, devices, etc-regardless of final outcome.

If the frequency of error is to be decreased, a clearer understanding of that process is needed. The circumstances that encourage error should be identified and the relative frequencies of different classes of errors should be established. While most anesthetists are aware of the potential for certain types of errors, corrective action is difficult to design or implement on the basis of anecdotal information. Established techniques exist for analyzing the etiology of error. However, they have not been previously applied to the practice of anesthesia. We have used a modification of the methodology known as criticalincident analysis to study preventable anesthetic mishaps. ${ }^{9}$ Although a somewhat subjective technique, it is a useful tool for transcending individual experience in a controlled manner, and is used often in human factors research for collecting information about human performance.

*This is a reprint of a paper that appeared in Anesthesiology, 1978, Volume 49 , pages 399-406.

\section{METHODS}

Information about preventable mishaps was collected by interviews with staff and resident anesthesiologists in one large metropolitan teaching hospital. A mishap was labeled a critical incident* when it was clearly an occurrence that could have led (if not discovered or corrected in time) or did lead to an undesirable outcome, ranging from increased length of hospital stay to death or permanent disability. In order to be included in the study, each incident was also required to have the following characteristics: (1) it involved an error by a member of the anesthesia team or a failure of the anesthetist's equipment to function properly; (2) it occurred at a time when the patient was under the care of an anesthetist; (3) it was described in clear detail by a person who either observed or was involved in the incident; (4) it was clearly preventable.† Outcome was recorded when possible but was not considered a criterion for inclusion of an incident. Despite use of these rules by the data analyst, no such limits were suggested to the interviewee in the course of the interview. Thus, interviewees reported whatever they felt constituted preventable error or failure.

The interviewees were selected at random from a list of departmental members and each was introduced to the study by a brief letter. All interviews were tape-recorded with the consent of the interviewees. They were conducted by the same interviewer, in the same neutral location, with a technique that had been developed and refined to minimize bias. Each interviewee was given a brief introduction outlining the general nature of the study, the population under study, the group

*As intended by Flanagan, the originator of the technique, the term "critical incident" refers to an occurrence that is significant or pivotal, in either a desirable or an undesirable way. For this study, we chose to examine only those incidents that had potentially undesirable consequences.

†Many complications of anesthesia are not "preventable" given the finite limits of medical knowledge. The incidents considered here are those where the anesthetist clearly failed to follow accepted practice or where a piece of equipment ceased to function normally. When doubt existed about preventability, the incident was excluded. 
conducting the study, and the anonymity and confidentiality with which reported data would be treated. The interviewee was then asked to describe preventable incidents that he or she had observed or participated in that involved either a human error or equipment malfunction.

The interviewer had extensive professional experience with interview technique and was trained in the rudiments of anesthesia practice. She was permitted to elicit details that were not volunteered in the interviewee's account of the incident. However, she was not allowed to suggest any specific type of occurrence. In some cases, where the interviewee had difficulty remembering incidents, the interviewer asked prompting questions, taken in order from a prepared list. These prompting questions were designed to be general and not suggestive of types of incidents, e.g. "Can you think of what might be called a 'typical' pitfall or recurrent problem in anesthesia?", followed by a request for an incident, when appropriate.

The interviews lasted 60 to 90 minutes, depending primarily upon the number of incidents the interviewee was able to recount. After completion of the interview, the interviewer prepared a condensed transcript from the tape recording. Details of each incident were then coded with a branching classification scheme. The coding of each incident was reviewed by a second investigator and, in cases of disagreement, by a third investigator. In all cases, incidents were coded according to the evaluation of failure made by the interviewee. Where this judgment was unclear, the incident was not included in the data base.

The classification was designed to describe each incident, including all circumstances that may have contributed to occurrence (table 1). Not all information could be obtained for each incident. Each of the 23 major categories of the classification included multiple branches for the observed varieties of data (fig 1). The data were first examined by generating summaries of the major categories. Questions of interest were posed and related data retrieved by selectively including or excluding categories or branches. The data were also examined by numerical sorting techniques in a search for non-obvious clusters of incident types and surrounding circumstances.

A series of 25 pilot interviews was conducted from January to May 1975 in order to evaluate interview strategies and to develop an interview format. The information collected relative to specific incidents was used to develop an initial version of the classification scheme and was not included in the data base. A second series of 47 interviews was conducted between September 1975 and April 1977 using the established interview format. The 47 interviewees included 11 staff anesthesiologists with ten or more years of practice each, 11 staff members with less than ten years of practice each, five third year residents, 16 second year residents, and four first year residents.

\section{RESULTS}

Three hundred and fifty nine incidents were identified and coded. There were 193 other reported occurrences that were transcribed but not coded because one or more of the criteria for qualification as an incident were not met. The staff reported an average of seven incidents per interview, while the average for residents was eight.* Fifty five per cent of all incidents reported had occurred within one year prior to the interview. Twenty per cent had occurred three years or more prior to the interview. Of the 359 incidents, 303 had occurred at the institution involved in the study. Fifty three reflected

*The range of reported occurrences per interview was 2 to 27 . The range of critical incidents extracted frorn these occurrences was 1 to 15 .
Table 1 The 23 major categories of information collected for each critical incident

Error or failure

Location of incident

Date of incident

Time of day

Hospital location

Patient condition before the incident

OR scheduling

Length of OR procedure

OR procedure

Anesthetic technique

Associated factors

Immediate consequence to patient

Secondary consequence to patient

Who discovered incident in progress

Who discovered incident cause

Discovery delay

Correction delay

Discovery of cause delay

Individual responsible for incident

Involvement of interviewee

Interviewee experience at time of interview

Related incidents

Important side comments

Table 2 The most frequent incidents*

\begin{tabular}{ll}
\hline Breathing circuit disconnection & 27 \\
Inadvertent gas flow change $\dagger$ & 22 \\
Syringe swap & 19 \\
Gas supply problem & 15 \\
Intravenous apparatus disconnection & 11 \\
Laryngoscope malfunction & 11 \\
Premature extubation & 10 \\
Breathing circuit connection error $†$ & 9 \\
Hypovolemia & 9 \\
Tracheal airway device position changes & 7
\end{tabular}

Tracheal airway device position changes

*This list includes both human error and equipment failures. Note that these somewhat arbitrary categories encompass only $39 \%$ of the total database, with the remainder representing a larger variety.

†See text for special nature of anesthesia machines involved.

experience of the interviewees at other institutions; locations of three incidents were not given.

The incidents ranged in seriousness from laryngoscope malfunctions, with no known consequence to the patient, to breathing-circuit disconnections that resulted in death. Human error was involved in $82 \%$ of the preventable incidents reported (fig 2) and equipment failure in $14 \%$ (fig 3 ). The remaining 16 incidents could not be clearly placed in either category, although they did fit the definition and criteria for inclusion in the study. All incidents involving disconnections were arbitrarily treated as human error. However, since the frequency of such disconnections is a direct consequence of the design of the connectors, these incidents could alternatively be considered equipment failures.

Ten categories of most frequently occurring incidents are listed in table 2. Syringe swap refers to incidents in which two syringes were inadvertently confused or interchanged and the wrong drug nearly (3 incidents) or actually (16 incidents) administered. Gas supply problems are difficulties or failures of the oxygen or nitrous oxide supply such as exhaustion of a cylinder's contents, inadvertent disconnection from a central gas supply, or a similar event. Premature extubation refers to cases in which a patient's trachea was extubated sooner than was clinically indicated and where the interviewee clearly described a failure to follow accepted practice (these were not cases involving subtle discriminations). Hypovolemia refers to incidents in which fluid replacement was not properly 


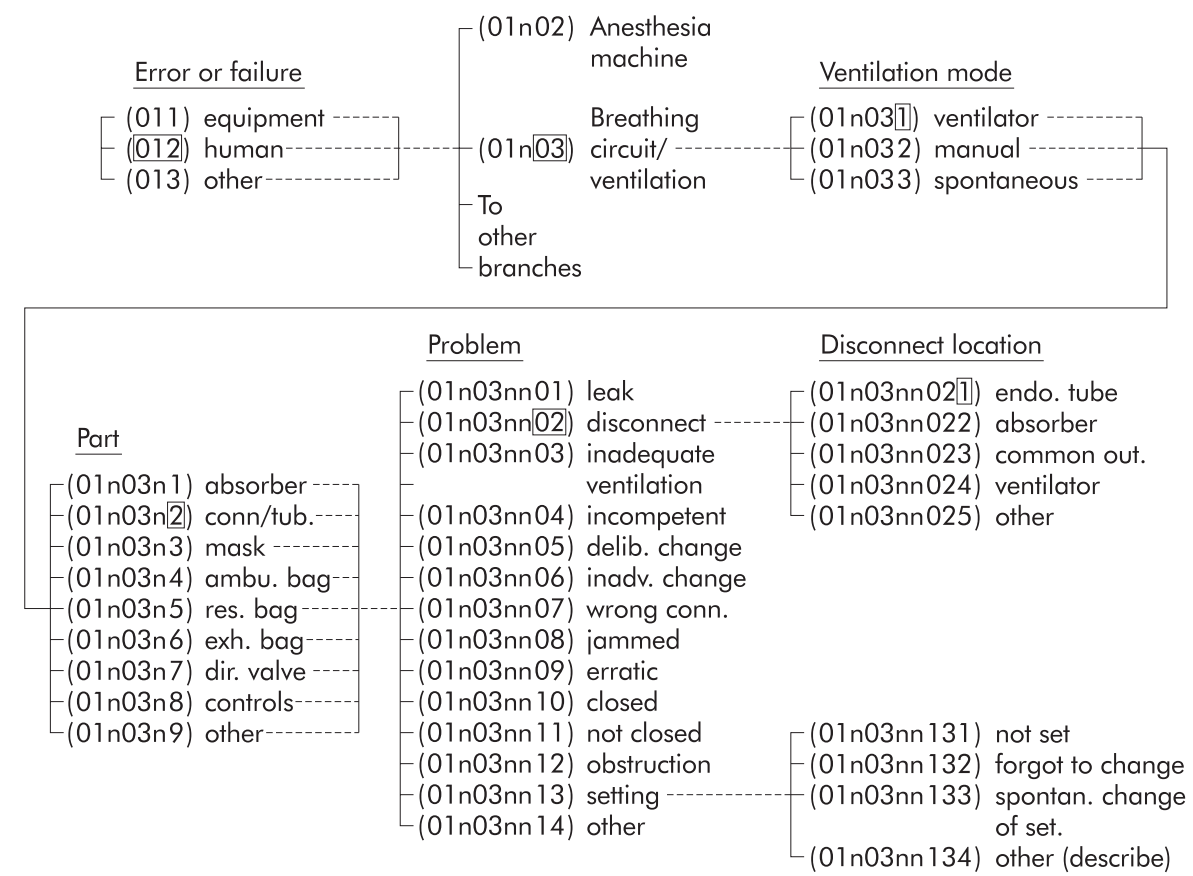

Figure 1 One of the many branches of one of the 23 major categories in the classification scheme. The material shown is approximately $4 \%$ of the entire scheme. The boxes represent a sample coding, that of an accidental disconnection in the breathing circuit at the endotracheal connector during mechanical ventilation. The numbers preceding the chosen cords or phrases generate a numerical string suitable for entry into a computerized data base. The string that describes this sample failure is 0120312021 . Twenty three such code strings were generated and entered for each incident included in the study.

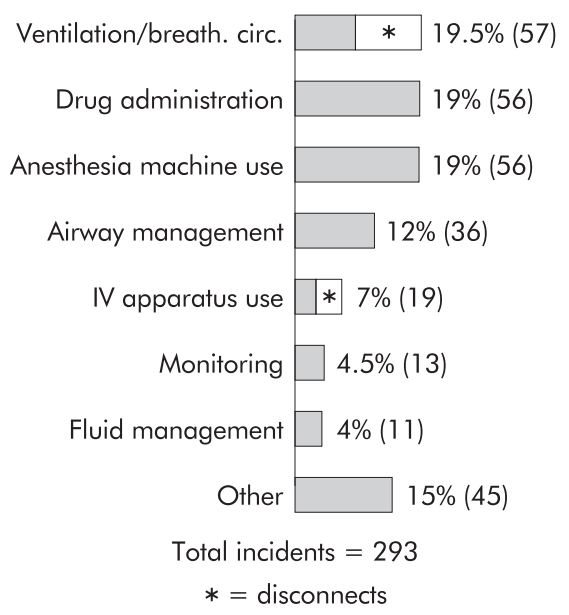

Figure 2 Distribution of human errors in subcategories defined by the nature of the activity. Thus, "anesthesia machine use" refers to errors primarily involving interaction with the machine. Numbers in parentheses represent actual numbers of incidents. The

disconnections of breathing circuit components and iv apparatus are distinguished by an asterisk, since their categorization as human errors rather than equipment failures is somewhat arbitrary.

managed, again, in the presence of unambiguous clinical indications as judged by the interviewee. The other incident types are self-explanatory.

Among the frequently occurring incidents, there were two types that were peculiar to the hospital studied. Several years ago, all anesthesia machines were modified by replacement of the oxygen flow control knob with a large, square, protruding, custom-made knob designed to provide obvious distinction by touch from the nitrous oxide knob. In addition, two years prior to the start of the study, all of the anesthesia machines were fitted with one particular type of gas scavenging device with an exhaust port that is adjacent to the reservoir bag

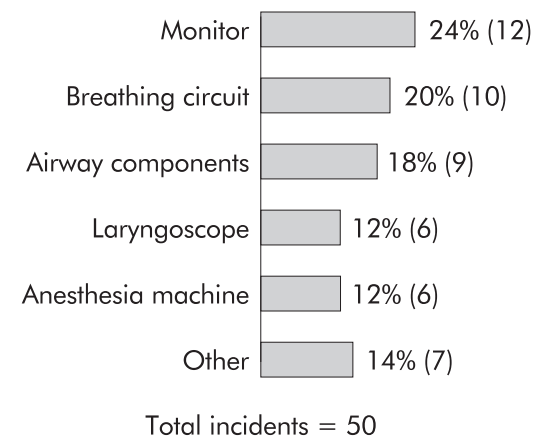

Figure 3 Distribution of equipment failures by subcategory.

connection and similar in shape. In eight of the 17 incidents in which the oxygen flow was inadvertently decreased, the cause was rotation of the square knob by impact from an object being placed on the machine's surface. Five of the nine incidents of a wrong connection in the breathing circuit involved transposition of the reservoir bag with the gas scavenging hose.

A category called "associated factors" was developed in response to descriptions in interviews. The category grew to include 44 possibly predisposing circumstances associated with incidents. There were 481 citations of such factors being in effect at the time of an incident, as stated or implied by the interviewee (table 3).

Critical incidents occurred most commonly during the middle of anesthesia and frequently during its induction (fig 4). The distribution of incidents by day (cases starting between 7:30 am and 5:00 pm) and night (all others) could be meaningfully summarized only for the final 19 interviews, in which a more deliberate effort was made to elicit this information. For those interviews, the assignment could be made for $91 \%$ of the incidents, with $79 \%$ of them occurring during the day and $21 \%$ at night. That distribution approximates the actual caseload distribution of the hospital ( $85 \%$ day, 15\% night). 
Table 3 Summary of associated factors cited

Inadequate total experience $\quad 77$

Inadequate familiarity with equipment/device

Poor communication with team, lab, etc

Haste

Inattention/carelessness

Fatigue

Excessive dependency on other personnel

Failure to perform a normal check

Training or experience-other factors

Supervisor not present enough

Environment or colleagues - other factors

Visual field restricted

Mental or physical-other factors

Inadequate familiarity with surgical procedure

Distraction

Poor labelling of controls, drugs, etc

Supervision - other factors

Situation precluded normal precautions

Inadequate familiarity with anesthetic technique

Teaching activity under way

Apprehension

Emergency case

Demanding or difficult case

Boredom

Nature of activity - other factors

Insufficient preparation

Slow procedure

Other

Total

77
45
27
26
26
24
24
22
22
18
18
17
16
14
13
12
12
10
10
9
8
6
6
5
5
3
3
3
481

Total incidents $=277$

Figure 4 Distribution of incidents with regard to stage of anesthesia delivery for induction and operating rooms.

It is possible to examine some individual types of incidents in a detailed way. As an example, a summary of some of the data associated with the 20 breathing circuit disconnections during mechanically controlled ventilation is shown in table 4 . Analysis to this depth for most errors or failures will require accumulation of more data.

\section{DISCUSSION}

This application of critical incident analysis to anesthetic practice has led to the development of an interview and coding technique useful for monitoring anesthesia mishaps. Although the kinds of incidents reported would probably be familiar to the experienced anesthetist, the technique offers a firmer basis for formulating and evaluating hypotheses about the etiology of anesthetic errors, and for designing and evaluating changes in training methods, procedures and equipment design. In this respect, it permits the application of human factors principles to anesthesia, following the example of successful applications in fields such as aviation.

There are many inherent documented problems associated with the use of retrospective interview techniques. ${ }^{10}$ The experimenter's dilemma is that any attempt to maintain a high degree of objectivity and reproducibility by asking

Table 4 Data associated with 20 incidents of breathing circuit disconnections during mechanically controlled ventilation

\begin{tabular}{ll}
\hline Location of circuit & 15 \\
Endotracheal tube connection & 5 \\
Other & \\
Time of day & 10 \\
Day & 1 \\
Night & 9 \\
Not recorded & \\
Anesthesia stage & 1 \\
Beginning & 18 \\
Middle & 1 \\
Not recorded & \\
Consequence to patient & 2 \\
Death & 3 \\
Arrest & 5 \\
Hypoxia & 10 \\
None & \\
Associated factors & 5 \\
Visual restriction & 3 \\
Fatigue & 3 \\
Distraction & 3 \\
Unfamiliarity with equipment & \\
Surgical procedure & \\
Gastrointestinal & 4 \\
Urologic & 3 \\
Neurologic & 5 \\
Not recorded & 2 \\
Other & \\
\hline
\end{tabular}

specific questions necessarily introduces bias. On the other hand, a completely non-directed interview decreases subject motivation and lowers the probability of obtaining pertinent information. In our earliest interviews, these extremes were intentionally explored. We then chose the following strategy for the interviews. No firm definition of an acceptable incident was presented to the interviewee in the introduction to the interview. No specific types of incidents were suggested. Details of incidents were, however, elicited with knowledgeable questions after the interviewee volunteered an initial description of an incident. He or she was not told how the data would be analyzed, and was merely informed that we were trying to learn more about preventable mishaps. The interviewer was not an anesthetist and was unknown to the interviewee prior to the interview.

This strategy served to collect incidents in a reasonably unbiased fashion with sufficient technical detail to allow qualified analysis. ${ }^{*}$ It is difficult to assess in any objective way the extent of residual bias. It is our opinion that the process was designed and executed with sufficient care to be effective for answering the qualitative questions we are asking of the data.

A non-judgmental and confidential atmosphere was deliberately created for these interviews. Interviewees were extremely cooperative, and the frankness of their reports may be indicated by the frequency with which they ascribed responsibility for errors to themselves ( 159 of the 255 human error incidents, exclusive of disconnections). Nonetheless, some inaccuracies in the data are inevitable due to the vagaries of recall. Given the qualitative objectives that characterize this phase of our studies, such inaccuracies can be tolerated in return for the larger quantity of incidents that are generated using a retrospective interview technique. The quality of the data could be improved by employing a prompt reporting method. ${ }^{11}{ }^{12}$

*We view the number of incidents retrieved in each interview as a sample representing an unknown fraction of the number actually experienced by the interviewee. Thus, only relative frequencies can be discussed. 
Most of the incidents reported in this study could be described as simply being "near misses." Indeed, 34\% (121/359) of the incidents had no reported effect on the patient, and only $17 \%(61 / 359)$ were known to have had more than a transient effect. Of these, there were 13 incidents that could be indicted as having contributed to mortality. Yet, we suspect that the factors inducing error are similar regardless of final outcome. Decreasing the frequency of occurrence for the entire set of errors should decrease it for the subset with unfortunate outcomes.

It is notable that, when given an unrestricted opportunity to discuss "pet peeves" in the form of incidents, anesthesiologists related their own errors more frequently than they did the "errors" of equipment or of other people. Although general complaints about equipment were frequently voiced, accounts of overt failures were relatively rare (50 incidents), and these only occasionally ( 9 of 50) resulted in sequelae lasting beyond the correction of the immediate problem. Of specific interest is that only six reported incidents involved the functional failure of an anesthesia machine (not including the breathing circuit), which may suggest a high degree of reliability or effective maintenance. (These machines received semi-annual preventive-maintenance inspections.) On the other hand, it is conceivable that anesthesia machines may be failing in ways that are not easily or rapidly detected by most anesthetists. In four of nine incidents of loss of oxygen supply in a machine equipped with a fail-safe system, the pressure fail-safe system was known to have been activated before the absence of oxygen flow was noticed by the anesthetist. Thus, at least one design approach for alerting the anesthetist to a potential problem appears to be a positive contribution.

Although the incidence of outright functional failure of equipment was low, machines can have shortcomings or faults in design by their propensity to encourage human error. Indeed, a substantial fraction of reported incidents of human error seemed to be related to the design or organization of equipment or devices, i.e. breathing circuit disconnections and misconnections, iv disconnections, inadvertent changes of gas flow, Copper Kettle control errors, and syringe swaps.

Although the number of incidents of any specific variety reported to date is relatively small, it is still interesting to consider possible explanations or corrective actions for those where a reasonable sample is available. The syringe swap was a frequently reported incident despite the fact that such occurrences are rarely described in the literature or mentioned in case discussion conferences in this particular institution. Although the reported consequences to the patients occurring as a result of these incidents were not serious, the potential for serious harm to the patient did exist for various periods in many of the incidents. There were distinct patterns that characterized the syringe swap incident. Relaxants or their antagonists were almost always involved (17/19), and the similarities of size, labeling or color were often (14/19) mentioned as a factor related to the error. Anesthesiologists usually devise their own systems for organizing their work spaces, and the motivation for doing so is typically personal experience with such an incident. The generic form of the problem is a familiar one to human factors specialists, and various labeling and packaging strategies are available to emphasize and reinforce the differences among various alternative controls or devices. A standardized system that would discourage such errors in the use of a set of drugs in syringes could be developed with relatively little difficulty, and would appear prudent.

The frequent reporting of breathing circuit disconnections with mechanical ventilators is probably not unexpected by most anesthetists. Examination of the circumstances surrounding such incidents (table 4 ) indicates that the outcome can be serious, the offending component is most often the endotracheal tube connector, and visual restriction, fatigue, distraction, and inadequate familiarity with certain ventilators may contribute to the probability of the occurrence. One can consider strategies for minimizing the likelihood of this type of incident, such as use of locking endotracheal tube connectors, use of integral disconnect alarm mechanisms for ventilators, and early training of residents in proper use of ventilators, the likelihood of disconnections, and appropriate precautions against their occurrence. Possible solutions should be carefully evaluated before general implementation in order to determine effectiveness.

The square oxygen knob and reservoir bag transposition incidents cited here are examples of errors peculiar to institutional practice. Yet they illustrate the need for evaluation before implementation. Procedures for controlling the kinds of equipment modifications that might lead to incidents of this type are often informal at most institutions. Under present conditions, many near misses and even serious incidents might occur before such situations were formally recognized and corrected.

We want to emphasize that the associated factors (table 3 ) were merely parts of the descriptions given by the interviewees. These factors did not necessarily induce errors, nor does their citation imply that they were invoked as excuses by interviewees. No clear relationship emerged between any specific factor and any specific type of incident. However, it was striking that the two most frequently occurring factors involved inadequate training or experience of the responsible anesthetist, who, in most cases, was a resident. Many of these incidents occurred despite the presence of what the resident felt was adequate supervision. Many are representative of the kinds of error that residents are considered prone to commit in the training process, e.g. premature extubation or anesthetic overdose, and that one hopes to preclude by close supervision. We are left with the impression that most of the errors and associated outcomes could be averted by a more structured approach to preparing residents for the environments into which they are often suddenly immersed. To the extent that certain kinds of errors are frequent, one could sensitize residents more effectively prior to involvement with certain devices or procedures.

In general, the reported errors and associated factors often related to training situations. Even where staff anesthesiologists reported their own mistakes (62 incidents), the incidents had often occurred earlier in their careers, when they were themselves residents (33 incidents). However, this statistic must be qualified by the observation that the staff members had spent most of their recent time in supervisory roles.

The anesthesia department involved in this study does not schedule relief from intraoperative anesthesia responsibilities, but it does allow its staff and residents to secure relief, when appropriate. In eight or nine incidents in which an anesthetist was being relieved, the replacement anesthetist discovered either the presence or cause of an incident in progress. In only one incident was the exchange of personnel a negative factor. Considering, in addition, that the reporting anesthetists mentioned fatigue as part of the incident description in 23 incidents and inattention or carelessness in 19 incidents, one could argue that a policy allowing relief for anesthetists is preferable to a policy that discourages relief.* The process of discovery of error deserves further study.

A pattern appeared in distribution of incidents with regard to the stage of anesthesia delivery. When prompted with the question, "At what stage of anesthesia is a problem most likely

*Preliminary results from a second hospital show similarities in most categories of incidents, but differ strikingly on the issue of relief. As many incidents were induced by the exchange of personnel as were discovered or corrected as a result of the relief process. It appears that transferences of responsibility for a case from one anesthetist to another in the two hospitals differ. Thus, providing relief may be a good practice, but only when carried out with careful transfer of information and authority. 
to occur," interviewees were usually of the opinion that induction or emergence, or both, were the most critical periods. Yet, nearly half of the incidents were reported to have occurred during the maintenance period (fig 4). This could mean that many errors occur, or proceed to a point where they are recognized as incidents, during the time when problems are least expected. The induction-emergence preconception may be a trap.

The decremental effect on vigilance of sustained observation over extended periods is a well established phenomenon in the human factors literature. ${ }^{13}{ }^{14}$ Work-rest cycles are commonly employed in many situations where the vigilance task is similar to that in anesthesia. Yet, there have been few, if any, attempts to apply this and other ergonomic concepts to clinical practice on a specialty-wide basis. ${ }^{15}{ }^{16}$

Some of the observations reported here have already been used to influence the human factors design for a new anesthesia delivery system. ${ }^{17}$ The methods described are now being applied at other hospitals to assess institutional differences and to develop the classification scheme further. A protocol is being evaluated for a prospective study to improve the quality of information collected and to prepare for a continuous monitoring program. From this sort of information, representing the compressed experience of a large set of anesthetists, can come the motivation and resolve to take specific actions and make specific investments to obviate some of the recurrent problems of anesthesia practice.

\section{ACKNOWLEDGEMENTS}

The authors gratefully acknowledge the contributions of Mr Edwin Trautman for designing the computer techniques used to store and analyze the data, Ms Elizabeth Silverberg for conducting some of the preliminary interviews, and Dr Melvin H Rudov for his advice on the experimental design. They offer special thanks to Dr Richard J Kitz for his support and encouragement. Deep appreciation is also due to the many anesthesiologists whose cooperation as participants in this study made it possible.

\section{Authors' affiliations}

J B Cooper, R S Newbower, C D Long, B McPeek, Laboratories of the Bioengineering Unit, Department of Anaesthesia, Harvard Medical School at the Massachusetts General Hospital, Boston, Massachusetts 02114 , USA

Supported in part by a grant from the National Institute of General Medical Sciences, GM 15904.

\section{REFERENCES}

1 Phillips OC, Capizzi LS. Anesthesia mortality. Clin Anesth 1974;10:220-44.

2 Dripps RD, Lamont A, Eckenhoff JE. The role of anesthesia in surgical mortality. JAMA 1961:178:261-6.

3 Clifton BS, Hotten WIT. Deaths associated with anesthesia. Br J Anaesth 1963;35:250-9.

4 Edwards G, Morton HJV, Pask EA, et al. Deaths associated with anesthesia: report on 1000 cases. Anaesthesia 1956;11:194-220.

5 Beecher HK, Todd DP. A study of the deaths associated with anesthesia and surgery based on a study of 599,548 anesthesias in ten institutions 1948-1952, inclusive. Ann Surg 1954;140:2-35.

6 Taylor G, Larson CP, Prestwich R. Unexpected cardiac arrest during anesthesia and surgery. JAMA 1976;236:2758-60.

7 Wylie WD. There, but for the grace of God . . . Ann R Coll Surg 1975;56:171-80.

8 Boba A. Death in the operating room. Springfield, IL: C C Thomas, 1965.

9 Flanagan JC. The critical incident technique. Psychol Bull 1954:51:327-58

10 Jung J. The experimenter's dilemma. New York: Harper and Row, 1971

11 Safren MA, Chapanis A. A critical incident study of hospital medication errors. Hospitals 1960;34:32-66.

12 Childs G. The critical incident study of surgical deaths and complication. Surgery in the United States, A Summary Report of the Study of Surgical Services for the United States, Volume 3. American College of Surgeons and American Surgical Association. Baltimore: Lewis Advertising Co, 1977: 2058-282.

13 Jerison HJ, Pickett RM. Vigilance: a review and reevaluation. Human Factors 1963;5:211-38.

14 Frankmann JP, Adams JA. Theories of vigilance. Psychol Bull 1962;59:257-72.
15 Blum LL. Equipment design and human limitations. Anesthesiology $1971 ; 35: 101-2$

16 Cooper JB, Newbower RS. The anesthesia machine: an accident waiting to happen. In: Pickett RM, Triggs TJ, eds. Human factors in healthcare. Lexington: Lexington Books, 1975: 345-58.

17 Cooper JB, Newbower RS, Moore JW, et al. A new anesthesia delivery system. Anesthesiology 1978;49:310-8.

\section{COMMENTARY}

\section{LOOKING BACK ON THE ANESTHESIA CRITICAL INCIDENT STUDIES AND THEIR ROLE IN CATALYSING PATIENT SAFETY}

In 1978 Cooper and colleagues published their landmark paper $^{1}$ on the application of the critical incident technique, adapted from uses in aviation and other fields, to examine the causes - and later prevention strategies-for adverse anesthesia outcomes. Following on 20 years of rudimentary anesthesia mortality studies, this was a brilliant approach that gave anesthesia clinicians new insights on which we could act. As the report told us: ". . factors associated with anesthetists and/or that may have predisposed anesthetists to err have, with a few exceptions, not been previously analyzed. Furthermore, no study has focused on the process of error - its causes, the circumstances that surround it, or its association with specific procedures, devices, etc-regardless of final outcome." Data from the study at one hospital and from an extension to four hospitals 6 years later ${ }^{3}$ provided two widely cited tables listing "the most frequent incidents" and "summary of associated factors cited". This innovative examination of critical events provided the first useful mirror for clinicians to reflect on their practice. In that mirror we were able to see how our imperfections could lead to errors and patient injury.

In my view this study was one of a few pivotal events responsible for the dramatic success in promoting anesthesia patient safety, starting with Beecher and Todd's paper on the rate of anesthesia mortality based on a large sample of operative cases. ${ }^{4}$ Later, in part catalysed by the reports from the critical incident studies, the Committee on Patient Safety and Risk Management in the American Society of Anesthesiologists (ASA) was created in 1984, the Anesthesia Patient Safety Foundation was founded in 1985, and the Closed Claim Analysis project was initiated by the ASA beginning in the mid 1980s.

Anesthesiology is the one domain in which patient safety was identified as a problem long before the Institute of Medicine's 1999 wake up call to the healthcare community. ${ }^{6}$ Not only was the problem identified in the late 1970s, but anesthesiologists faced the issues, taking actions to effect changes that would reduce errors, adverse outcomes, and injuries. While it is often difficult to trace the historical path of change, there is reason to believe that the anesthesia critical incident studies planted seeds of ideas for others, either directly or subliminally. The studies demonstrated the power of qualitative research (although that term was not used at the time). They catalysed change in anesthesia. In combination with the influences noted above, they fostered the strong culture of safety for which anesthesiology is recognised.

The first peer reviewed publication of the critical incident studies "Preventable anesthesia mishaps: a study of human factors" $^{\prime 1}$ is reproduced in this issue of QSHC. Several other papers appeared over the next few years, the most widely cited of which is the 1984 study entitled "An analysis of major errors and equipment failures in anesthesia management: considerations for prevention and detection" which also appeared in Anesthesiology. These papers describe a then almost unknown methodology for medicine, although it had been applied in two prior studies, one of hospital medication 
error (in 1960) ${ }^{7}$ and the other to the beneficial and detrimental performance of physicians from several specialties (in 1970). ${ }^{8}$ Yet, neither of those studies inspired the kind of action of the reports in Anesthesiology. Perhaps it was the way the method was used to examine the underlying mechanisms of error or just a matter of timing that gave the anesthesia studies their enormous impact. For whatever reasons, anesthesia clinicians gained the insights needed to effect change in their basic attitudes and practices toward dealing with errors.

The critical incident technique is now used more often, but still not as often as it might be in this new era of interest in errors and system failures. As applied by Cooper et al, the approach was employed to capture first hand reports of human errors and equipment failures that had actual or potential negative consequences. The definition they crafted for a critical incident has been revised in many ways over the years in studies of adverse events, but the main wording of it is still generally applicable: "A mishap was labeled a critical incident when it was clearly an occurrence that could have led (if not discovered and corrected in time) or did lead to an undesirable outcome, ranging from increased length of hospital stay to death or permanent disability".

Using an inductive approach with well specified definitions, the investigators analysed the events in search of the underlying causes of error and what we would now call "failure to recover" from error. Later, deeper analysis identified practical understandable strategies that would prevent or trap most errors. Today the thinking about human error and systems failures is more sophisticated and the methodologies to study errors are more rigorous (most would seek inter-rater reliability rather than a consensus approach to rating incidents). Yet, for the study of critical events, both positive and negative, the critical incident approach is still attractive. Now aided by then unimaginable computer power and web access and by qualitative analysis software, it is a method that many can apply to explore patient safety questions in all medical fields. On the other hand, such studies have become more difficult to undertake because of the barriers created by mandatory reporting requirements for errors reported even in research studies.

These original studies were emulated by anesthesiologists in several countries, most notably by Williamson et al in Australia. ${ }^{9}$ That paper was itself the catalyst for one of the earliest and most informative national incident reporting systems, the Australian Incident Monitoring System (AIMS). ${ }^{10}$ Other investigations using the critical incident technique had an impact in their native countries, contributing greatly to the development of standards of practice which are now essentially universal.

The critical incident studies were elegant in their simplicity. While primitive in some respects, the findings are still relevant today. With the benefit of time it may seem obvious that such research and methodology were needed to expose a hidden truth that had been kept by health care for many years. But, recall that there was little before to prompt this kind of research. Thus, by their pioneering innovative approach to examine a previously unidentified problem, tactful and eloquent word crafting, and sensitivity to what clinicians needed to know about themselves, Cooper and his colleagues have made an immeasurable contribution to anesthesia. By the road they paved, that contribution has been applied to all of health care.

E C Pierce Jr 770 Boylston St \# 10C, Boston, MA 02199 , USA; epierce500@aol.com

\section{REFERENCES}

1 Cooper JB, Newbower RS, Long CD, et al. Preventable anesthesia mishaps: a human factors study. Anesthesiology 1978;49:399-406.

2 Flanagan JC. The critical incident technique. Psychol Bull 1954:51:327-58.

3 Cooper JB, Newbower RS, Kitz RJ. An analysis of major errors and equipment failures in anesthesia management: considerations for prevention and detection. Anesthesiology 1984;60:34-42.

4 Beecher HK, Todd DP. A study of the deaths associated with anesthesia and surgery. Ann Surg 1954;140:1-34.

5 Cheney FW, Posner K, Caplan RA, et al. Standard of care and anesthesia liability JAMA 1989;261:1599-603.

6 Kohn IT, Corrigan JM, Donaldson MS, eds. To err is human: building a safer healthcare system. Washington DC: National Academy Press, 1999.

7 Safren MA, Chapanis A. A critical incident study of hospital medication errors. Hospitals 1960;53-8

8 Sanazarro PJ, Williamson JW. Physician performance and its effects on patients: a classification based on reports by internists, surgeons, pediatricians and obstetricians. Med Care 1970;4:299-308.

9 Williamson JA, Webb RK, Pryor GL. Anesthesia safety and the "critical incident" technique. Aust Clin Rev 1985;17:57-61.

10 Webb RK, Currie M, Morgan CA, et al. The Australian Incident Monitoring Study: an analysis of 2000 incident reports. Anaesth Intens Care 1993;21:520-8 (and entire issue of journal). 